\title{
PREVALENCIA DE CANDIDA ALBICANS EN INDIVIDUOS PORTADORES DE PRÓTESIS TOTAL QUE ASISTEN A LA CLÍNICA ODONTOLÓGICA DE L.A UNIVERSIDAD SANTO TOMÁS Y A INSTITUCIONES GERIÁTRICAS DE FLORIDABLANCA
}

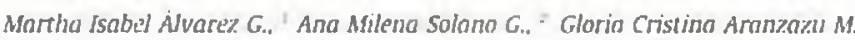
Estudiante de $X$ semestre, Facultad de Odonologia, U. Santo Tomis.

'Odontologa. U. Santo Tomís. Fspecialista en Putologin y Medios Diagnósticos. U. Q B Bosque. Docente U. Santo Tounós

Autor n'sponsable de correspondencio: Gloria Cristina Aranzazu M.

Correo electronica: aranzazugloria (a yahoo.es

\section{RESUMEN}

Objetivo: Identilicar la frecuencia de Candida albicans en pacientes portadores de prótesis total con o sin signos y sintomos clinicos evidentes que asisten a las Clinicas Odontológicas de la Universidad Sonto Tomäs y a instiluciones geriátricas de Floridablanca en el periodo de un año.

Materiales y métodos: Se realizó un estudio observacional analitico de corte transversal, en adultos mayores. Se recolectó información relacionada con antecedentes clinicos en cavidad oral y variables sociodemográficas generales. La prevalencia de Candida albicans se determino mediante prueba de tubo germinal, azul de lactofenol e hidróxido de potasio, se aplicaron pruebas estadisticas como chi" y test exacto de Fisher.

Resultados: Se tomaron 70 muestras. 47 de ellas en pacientes con signos y sintomas de candidiasis (67.1\%), 38 localizadas en paladar, siendo el eritema de paladar el más frecuente en un 37.19n. Las pruebas de laboratorio de azul de loctofenol (ALF) e hidróxido de potasio $(\mathrm{KOH})$ resultaron ser especificas pero no lo suficientemente sensibles. Por el contrario. Ja prueba de tubo germinal demostró ser positiva en 47 muestras con una prevalencia de (67.14\%) de los pacientes en muestras de mucosa oral y $53(75.71 \%)$ en muestras de prötesis.

Conclusiones: Se encontrú una prevalencia importante asuciada con el uso de prótesis total y con la presencia de signos y sintomas, especialmente. lesiones rojas de la mucosa palatina lo que sugiere una relación importante con el diagnóstico de estomatitis sub protésica. [Álvarez MI. Solano AM. Aranzazu G. Prevalencia de Condida albicans en individuos portadores de prótesis total que asisten a la clinica odontológica de lo Universidad Santo Tomás y a instituciones geriátricas de Floridablanca. Ustasalud Odontologia 2005; 4: $17 \cdot 221$

Palabras clave: Condida albicans, Prótesis, Estomatitis.

\section{PREVALENCE OF CANDIDA albicans in SUBJECTS WITH DENTAl PROSTHESIS THAT ATTEND THE DENTAL CLINICS OF THE SANTO TOMAS UNIVERSITY AND GERIATRICAL INSTITUTIONS FROM FLORIDABLANCA}

\begin{abstract}
objective: To identify the frequency of Candida albicans in potients with dental prosthesis with or without signs and evident clinical symptums those attend the Dental Clinics of the Santo Tomas University and geriatric institutions of Floridablanca in the period of a year.

Materials and methods: An analytical observational study of cross section was made. The collected information related to clinical background in oral cavity and general demographics variables. The prevalence of Condida albicans was determined by means of the test of germinal. blue tube of lactofenol and hydroxide of potassium. to statistical analysis like chi2 and exact test of Fisher were applied.

Results: Seventy samples, 47 of them in potients with signs and symptons of candidiasis were taken (67.1\%), 38 located in palate, being eritema of palate most frequent in a $37.1 \%$. The laboratory lests of blue of lactofenol (ALF) and hydroxide of potassium $(\mathrm{KOH})$ turned out to be specific but not it sufficiently sensible. On the other hand, the germinal tube test demonstrated to be positive in 47 samples with a prevalence of $(67.14 \%$ ) of the patients in samples of oral mucosa and 53 (75.71\%) in prosthesis samples.

Conclusions: It was an associate important prevalence with the use of total prosthesis and the presence of signs and symptoms. specially, red injuries of the palatal mucosa thar suggests an imponant relation with the diagnosis of prosthesis stomatitis.
\end{abstract}

Key words: Candida albicans, Prosthesis, Stomatitis. 


\section{INTRODUCCIÓN}

Se han descrito más de 100 especies distintas del género Candida, todas ampliamente distribuidas en la naturaleza. Estas levaduras pueden estar formando parte de la microbiota normal de la cavidad oral (lengua, paladar. mucosa oral), tubo digestivo (estómago, intestino) y vagina (al menos en el 30\% de las mujeres).' 'Sólo bajo determinadas circunstancias dan origen a lesiones y procesos diversos, de carácter agudo, subagudo o crónico, englobados en el término de candidiasis o monoliasis. ${ }^{20}$

Generalmente, el paciente con candidiasis tiene alteraciones fisiológicas y de orden inmune que comprometen la integridad de los tegumentos, alteran la flora normal 0 impiden la normal expresión de la inmunidad. Hay ciertos factores locales y sistémicos que favorecen el desarrollo de candidiasis oral manifestandose como estomatitis sub protésica (EPD). ${ }^{8.9}$ Dentro de éstos factores se pueden mencionar el uso de acondicionadores de tejidos. adhesivos protésicos, xerostomía, desadaptación de las prótesis que permite un desbalance oclusal lo que favorece la aparición de áreas traumáticas sobre la mucosa de soporte e hipersensibilidad a los materiales de confección de la prótesis."'12

Investigadores como Dar-Odeh encontraron que Candida albicans era la responsable de la mayoria de estomatitis relacionadas con prótesis $(72 \%)$ y también fue la única especie secretora de proteinasas asparticas capaces de dañar la integridad de la mucosa. ${ }^{13.14}$

Debido a la presencia de este agente en el organismo, el diagnóstico de candidiasis debe ser estructurado conjuntamente con la identificación de Candida albicans. Un diagnóstico de presunción se realiza por la observación microscópica de abundantes formas levadurifomes y pseudohifas en el material examinado; comúnmente se emplea la observación en fresco con adición de solución de potasio (KOH) o el azul de lactofenol. El cultivo se practica sobre el agar de sabouraud; la incubación puede hacerse a temperatura ambiente o preferiblemente a $37^{\circ} \mathrm{C} .13$

La identificación de levaduras es, relativamente, rápida en el caso de Candida albicans ya que se realiza por medio del test tubo germinativo una vez han sido aisladas (incubación a $37^{\circ} \mathrm{C}$ durante 24 - 48 horas). En caso de ser negativo, es decir, de no ser Candida albicans, es necesario utilizar técnicas fisiológicas y bioquímicas que con- sisten en el estudio del patrón de asimilación de azucares. Si bien en este caso, el tiempo de procesado es mayor retrasándose los resultados, pueden ser útiles para el clinico dada la resistencia observada en ciertas especies del género Candida a los derivados inmidazólicos. Técnicas comercializadas como Fongiscreen $4 \mathrm{H}^{\circledast}$ y Rapidec albicans ${ }^{\circledR}$ permiten una identificación rápida de levaduras mediante el método enzimático que, tambièn, se pueden conseguir con base en cambios de color en el medio de cultivo de distintas especies del género Candida, como albicans ID ${ }^{\circledast}$ y CHROMagar Cándida ${ }^{\circledR}{ }^{3}$

El propósito de este estudio fue conocer la prevalencia de Candida albicans en individuos portadores de protesis total que asistion a las Clinicas Odontológicas de la Universidad Santo Tomás y a instituciones geriátricas de Floridablanca.

\section{MATERIALES Y MÉTODOS}

Se realizó un estudio de corte transversal con 70 pacientes usuarios de prótesis total (41 mujeres y 29 hombres) que asistieron a las Clinicas Odontológicas de la Universidad Santo Tomás y a instituciones geriátricas de Floridablanca de enero a diciembre de 2003. La muestra se calculó teniendo en cuenta un nivel de confianza del $95 \%$, un poder del $80 \%$ y una prevalencia de candidiasis en usuarios de prótesis del $60 \%$. Se incluyeron individuos usuarios de prótesis total, minimo con un año de uso; se excluyeron individuos con enfermedades sistémicas como diabetes mellitus y enfermedades carenciales e inmunosupresoras, con ingesta de medicamentos (antibióticos, antifúngicos, corticoides, antineoplásicos), e individuos con alteraciones metabólicas como hipertiroidismo, hiperparatiroidismo e hipotiroidismo, por el riesgo que tienen de presentar candidiasis debido a su estado inmunológico.

Se informó sobre los objetivos del estudio y se firmó una carta de consentimiento informado, respetando los principios éticos como autonomia y beneficencia. La resolución 008430 del Ministerio de Salud del 93 considera este estudio como de riesgo minimo y recibió aprobación del Comité de Ética Institucional.

El estudio fue de tipo observacional y descriptivo con el fin de determinar la prevalencia de Candida albicans. Se registraron variables sociodemográficas como género. edad, escolaridad y estrato socioeconómico. 
El género se registró como variable dicotómica medida en escala nominal como masculino o femenino: la edad se registró en escala de razón como número de años cumplidos, variable continua. Por otra parte, la escolaridad se registró en escala de razón como número de años de estudio. variable continua; el estrato socioeconómico se registró en escala de razón. variable continúa; los años de uso se registraron en escala de razón como número de años, variable continúa, y las horas de uso diario se registraron en escala de razón como numero de horas, variable continua.

Otras variables fueron antecedentes clínicos de la mucosa palatina o reborde alveolar, síntomas como prurito y quemazón y signos como hiperplasia y eritema. Además, del antecedente clínico de la prótesis total superior o inferior que se registraron en forma dicotómica en escala nominal como si (1) o no (0). Se tuvo en cuenta la localización de la lesión (superior o inferior), los sintomas y la condición de la prótesis.

Se establecieron varios horarios con el fin de evitar sesgos en las técnicas de identificación y cultivo, y en la toma de las muestras. Una persona tomó las muestras en un lugar especifico. Adicionalmente, se ofrecieron los servicios odontológicos básicos gratuitos como examen clínico - odontológico completo.

I.as muestras de la prótesis y de la mucosa se tomaron simultáneamente con un aplicador estéril para realizar un adecuado barrido de la superficie tanto de la mucosa como de la prótesis. Se les realizó el examen directo de $\mathrm{KOH}$, y cultivos en Agar Sabouraud que fueron llevados a la incubadora por 48 horas a $37^{\circ} \mathrm{C}$; se les aplicó tinción con Azul de Lactofenol. Los cultivos positivos se confirmaron con la prueba del tubo germinal, prueba especifica para Candida albicans. Los cultivos positivos fueron comparados con la cepa de referencia ATCC 18804. Posteriormente los resultados se llevaron a una base de datos en Excel y se realizó el análisis en STATA 6.0. ${ }^{15.16}$

\section{Lectura de las placas}

En algunas placas se detectó la presencia de levaduras. blastoconidias y pseudomicelios en microscopio de luz con un aumento de $100 \mathrm{X}$.

\section{Lectura de los cultivos}

A las 48 horas de cultivadas las muestras se determinó la presencia de Candida por las colonias observadas, que se presentaron en forma de gota de leche, bien definidas y de aspecto musgoso.

\section{RESULTADOS}

De la población estudiada, 47 (67.1\%) presentaron lesión clinica evidente en cavidad bucal que sugería la presencia de Candida, 16 de éstas (22.9\%) fueron de lesión blanca (Tabla 1).

Del total de los que presentaron lesiones, 26 (37.1\%) fueron por eritema en el paladar que fue el signo más frecuente y $10(31 \%)$ fueron lesiones blancas en la misma región (Tabla 2).

De la población examinada, presentaron un promedio de uso diario de la prótesis dental de 21.45 horas y un uso en años de 7.75 (Tabla 3).

Se realizaron pruebas de laboratorio como cultivo, $\mathrm{KOH}$, azul de lactofenol y tubo germinal, se observó la frecuencia de Candida albicans en prótesis y mucosa oral. El $67.1 \%$ fue tubo germinal positivo en mucosa y un $75.7 \%$. tubo germinal positivo en prótesis (Tabla 4).

De los que presentaron tubo germinal positivo, 34 (72.3\%) presentaron signos y sintomas en mucosa, y $38(80.8 \%)$ de quienes presentaron signos y síntomas fueron TGP en prótesis; de éstos, 26 (70.3\%) presentaron eritema con TGP en mucosa, y 31 (83.8\%) TGP en prótesis con una significancia marginal de 0.095 y se presentó con mayor frecuencia eritema en el paladar, $24(75 \%)$ muestra de mucosa, y $26(81.2 \%)$ de prótesis (Tabla 5).

Al comparar la sensibilidad y la especificidad de la prueba de $\mathrm{KOH}$ y azul de lactofenol, comparadas con la prueba de tubo germinal en la determinación de Candida albicans, queda claro que los niveles de sensibilidad son bajos en $\mathrm{KOH}, 1(8.5 \%)$ en mucosa y los niveles de especificidad son altos, 23 (95.7\%) en mucosa (Tabla 6).

\begin{tabular}{lccl}
\hline \multicolumn{1}{c}{ SIGNO ORAL } & FRECUENCIA & $|\%|$ \\
\hline Presencia de lesiones & 47 & 67.1 \\
Hiperplasia & 4 & 5.7 \\
Lesión blanca & 16 & 22.9 \\
\hline
\end{tabular}

Tabla 1. Descripción de la población según signos orales.

n: 70, algunos sujetos presentaron más de un signo

\begin{tabular}{lccc}
\hline & $\begin{array}{c}\text { HIPERPLASIA } \\
\text { Frecuencia (\%) }\end{array}$ & $\begin{array}{c}\text { ERITEMA } \\
\text { Frecuencio (\%) }\end{array}$ & $\begin{array}{c}\text { LESIÓN BLANCA } \\
\text { Frecuencia (\%) }\end{array}$ \\
\hline Paladar & $2(2.9)$ & $26(37.1)$ & \\
Reborde & $1(1.4)$ & $3(4.3)$ & $8(11.4)$ \\
Surco & $1(1.4)$ & $8(11.4)$ & $4(5.7)$ \\
\hline
\end{tabular}

Tabla 2. Descrippción de las lesiones según su localización. 


\begin{tabular}{lcc}
\hline \multicolumn{1}{c}{ VARIABLE } & PROMEDIO & D.E."* \\
Añas de usa & 7.75 & 6.33 \\
Uso diario (horas) & 21.45 & 3.74 \\
Lavados diarios (veces al dia) & 2.38 & 0.82
\end{tabular}

Tabla 3. Descripción de usa y frecuencia de higiene de la prótesis.

п:70 •Promedio "a DE: Desviación estóndar

\begin{tabular}{lcc}
\hline PRUEBA DE LAB. & $\begin{array}{c}\text { MUcOSA } \\
\text { Frecuencia }(\%)\end{array}$ & $\begin{array}{c}\text { PRÓTESIS } \\
\text { Frecuencia (\%) }\end{array}$ \\
\hline Tubo germinal & $47(67.14)$ & $53(75.71)$ \\
KOH & $5(7.14)$ & $20(28.57)$ \\
ALF. & $1(1.43)$ & $28(40)$ \\
\hline
\end{tabular}

Tabla 4. Frecuencia de Candida albicans según la prueba de Laboratorio.

n. 70 KOH: hidróxidu de potusso - ALF: azul de lactofenol

\section{DISCUSIÓN}

Candida albicans es un microorganismo habitante normal de la cavidad bucal, puede ocasionar enfermedades clínicas particularmente pacientes con diabetes mellitas (S. Sjögren's), inmunosupresión y otros. ${ }^{[.7,4,13.17 \text {, in }}$

El uso de prótesis total se ha considerado como un factor facilitador para la presencia de Candida albicans y por lo tanto asociada a la mayoría de mucositis o estomatitis por prótesis total diagnosticada por el odontólogo. 5.17

Se observaron 70 pacientes usuarios de prótesis total superior, a quienes se les examinó por la presencia de lesiones clinicas asociadas a candidiasis como eritema, lesión blanca e hiperplasia y otras. Se tomaron muestras para cultivo de prótesis total y de la mucosa oral tanto

\begin{tabular}{lcc}
\hline & MUCOSA & PRÓTESIS \\
\hline KOH* & & \\
Sensibilidad & $8.5 \%(1)$ & $35.8 \%(19)$ \\
Especificidad & $95.7 \%(23)$ & $94.1 \%(16)$ \\
VPP** & $80 \%(1)$ & $95 \%(19)$ \\
VPN & $32 \%(16)$ \\
ALF*. & $33.8 \%(23)$ & \\
Sensibilidad & $2.1 \%(1)$ & $35.8 \%(19)$ \\
Especificidad & $100 \%(23)$ & $94.1 \%(16)$ \\
VPP *. & $100 \%(1)$ & $95 \%(19)$ \\
VPN & $33 \%(23)$ & $32 \%(16)$ \\
\hline
\end{tabular}

Tabla 6. Niveles de sensibilidad y especificidad, valor predictivo positivo y negativo de las pruebas de $\mathrm{KOH}$ y ALF de muestras aisladas de mucosa y prótesis al comparar con pruebas de tubo germinal.

ก:70 ' KOH: hidróxido de potasio. "AlF: azul de lactofenol.

". VPP: valor predictivo postivo m- VPN: valor predictivo negativo

en pacientes con y sin signos y sintomas. De los pacientes con eritema, el $83.8 \%$ presentaron tubo germinal positivo de la muestra tomada de la prótesis, similar a observado por Mejía, López y Uribe en 1991. 2" Sin embargo, cuando la muestra se tomó en mucosa oral se encontró una frecuencia de $70,3 \%$, esto se puede explicar por la adherencia de Candida albicans a la superficie de la prótesis, como lo corrobora Valencia en $1989 .{ }^{19}$ y McCourtier y Douglas en 1984."

Se encontró una frecuencia de tubo germinal positivo de $75.71 \%$ entre los pacientes que lavan la prótesis y la usan continuamente lo que se asocia con factores coadyuvantes a la presencia de Candida al igual que lo identifican Mejia, López y Uribe, y Poirier y colaboradores quienes establecen que la virulencia de Candida albicans es consecuencia de su persistencia sobre la superficie de la mucosa favorecida por la prótesis. $10,14.211$

\begin{tabular}{|c|c|c|c|c|}
\hline VARIABLE & $\begin{array}{c}\text { TUBO GERMINAL + } \\
\text { MUCOSA } \\
\text { Frecuencia (\%) }\end{array}$ & $\mathbf{P}$ & $\begin{array}{l}\text { TUBO GERMINAL + } \\
\text { PRÓTESIS } \\
\text { Frecuencia (\%) }\end{array}$ & $\mathbf{P}$ \\
\hline Signos y sintomas & $34(72.3)$ & $0,186^{\circ}$ & $38(80.8)$ & $0,152^{\circ}$ \\
\hline Hiperplasia & $3(75)$ & $0,601^{* *}$ & $3(75)$ & $0,681^{* *}$ \\
\hline Eritema & $26(70.3)$ & $0,555^{\circ}$ & $31(83.8)$ & $0,095 . . \cdots$ \\
\hline Lesión blanca & $13(81.2)$ & $0,232^{\circ}$ & $13(81,2)$ & $0,744^{\circ}$ \\
\hline \multicolumn{5}{|l|}{ Localización } \\
\hline Paladar & $24(75)$ & $0,245^{\circ}$ & $26(81,2)$ & $0,652^{\circ *}$ \\
\hline Reborde & $6(85.7)$ & & $6(85.7)$ & \\
\hline Surco & $3(42.9)$ & & $5(71.4)$ & \\
\hline
\end{tabular}

Tabla 5. Descripción de signos y sintomas según presencia de tubo germinal positivo. n: 70 . Test de chi ${ }^{2}$ ". Test exacto de Fisher … Significancia marginal $\propto: 0.05$ 
Se han determinado algunos factores predisponentes como la desadaptación protésica. ${ }^{[321}$ Este estudio no permite demostrar esa asociación; veintiocho pacientes $(77.8 \%)$ con prótesis adaptadas presentaban tubo germinal positivo en muestras de mucosa y $30(83.3 \%)$ en la muestra de la prótesis, lo que no permite sugerir esta asociación.

Rodriguez, Miranda y colaboradores establecieron que un $70 \%$ de individuos con signos clínicos de estomatitis presentaron Candida albicans. ${ }^{13}$ En este estudio se encontró que un $34(72.3 \%)$ de los individuos con signos y sintomas presentaban tubo germinal positivo en mucosa, y $38(80.8 \%)$ en prótesis, lo que corrobora dichas apreciaciones, igualmente establecidas por McMullan-Vogel y colaboradores, y por Dinatale en 1999. ${ }^{15,22}$

I.a confirmación de la presencia de Candida albicans con tubo germinal fue realizada por Dinatale $(1999)^{2 n} \mathrm{y}$ por Darwazeb y colaboradores (2001). . $^{21}$ mediante la observación de las colonias es imposible su diferenciación con otras especies. Además, en sus estadios demuestran una mayor afección por el género femenino.

I.a prueba directo de $\mathrm{KOH}$ y el azul de lactofenol, no fueron lo suficientemente especificas en la identificación de Candida albicans.

El $52.56 \%$ de los pacientes sin lesión clínica presentaron tubo germinal positivo, lo que confirma que Candida albicans es un habitante normal de la cavidad bucal y con la presencia de otros factores predisponen a lesiones y a diversos procesos patológicos. ${ }^{2.11}$

\section{CONCLUSIONES}

Candida albicans es un habitante normal de la cavidad bucal que necesita de condiciones especiales para producir la enfermedad.

- Factores relacionados en la teoría con signos y síntomas de candidiasis como desadaptación de prótesis no se relacionaron en este estudio con la presencia de Candida.

- La adaptación de la prótesis favorece el contacto prolongado y la adherencia de Candida albicans a la mucosa y esto asociado a una pobre higiene y uso prolongado favorece aún más la aparición de signos y sintomas. ${ }^{12,21}$
* Las lesiones más comunes asociadas con presencia de Candida albicans son las lesiones rojas y la localización más común es el paladar.

- Las pruebas de $\mathrm{KOH}$ y ALF no son lo suficientemente sensibles pero si específicas para ser utilizadas en el diagnóstico de Candida albicans en pruebas de tamizaje.

\section{BIBLOOGRAFIA}

1. Liebana J. Microbiolugia oral. México. McGraw.Hill Interamericana Editores S.A. p. 370 -375. 544 - 552 .

2. Divo A. Microbiologio mèdica. México. Nueva Editorial Interamericana S.A. p.369 - 373 .

3. Corrillo A], Tur-Tur C. Mongos dermatofitos: Aspectos biológicos. Actualidad dermatológica. 1995; p. 699 - 706.

4. Fuentes B, Garcio Vidal I. Factores predisponentes sistémicos de la candidiasis oral. En: Medicina general 2002. 41. p.121-125.

5. Rincón S. Guia para el curso teórico de micologia. B/manga. Editorial 1987. p. $36 \cdot 37,146-148$.

6. Mirvik Q. Pearsall N. Weisser R. Bacteriologia y micologio médicas. México. Nueva liditorial Interamericana S.A. p. 459 - 465.

7. Restrepo A. et al. Fundamentos de Medicina. Fnfermedades Infecciosas. Medellin-Colombia 1996. Editorial CIB $5^{9}$ Edición. Capitulo Condidiasis. p. 265 - 275.

8. Franks A. Hedegard B. Odontologia geriátrica. Barcelona 1976. Editorial Labor. S.A. p. $137 \cdot 138$.

9. Abraham C, Al-Hashimi 1, Haghighat N. Kvaluation of the levels of oral Candida in patients with Sjogren's syndrome. Oral Surg Oral Med Oral Pathol 1998; 86: 65 - 67.

10. Poirier AC, Chinenos E. Ferrer M. López. J. Caballero R. Importancio de los factores predisponentes en la candidiasis bucal. Medicinn oral 1997: 2: $21-29$.

11. Peman J. El papel del microbiólugo clinico en las condidosis sistémicas. URL disponible en http:www.seimc.org/control/revi_Mico; pdficripto.pdf

12. Mccourtie J, Douglas LJ. Relationship between cell surface composition of Candida albicans and adherence to acrylic after growth on different carbon sources. Infect Immun 1981: 32: 1234 - 1241.

13. Rodrigue\% J. Miranda J. Morejon H. Santana J. Candidiasis de la mucosa bucal. Revisión bibliográfica. Rev Cubana Estomatol 2002; 39: $187-233$. 
14. Dar-Odeh NS, Shejabi AA. Oral candidiasis in patients with renovible dentares. Mycoses 2003; 46: 187.

15. Microsoft Excel 5.0, Microsoft Corporation 1997.

16. Stata Corporation 1999. Stata Statistical Software. Release 6.0. College Station TX: Stata Corporation.

17. McMullan-Vogel CG, Jude HD, Ollert MW, Vogel CW. Serotype distribution and secretory acid proteinase activity of Candida albicans isolated from the oral mucosa of patients with denture stomatitis. Oral Microbiol Inmunol 1999; 14: 183 - 189.

18. Conant N, Tillerson D, Baker K. Micología. México. Interamericano S.A. 1972. p. $264 \cdot 279$.

19. Valencia SB. Adherencia de la Candida albicans a la prótesis de acrílico utilizada en nuestro medio. Revista CES Odontología 1989: 2: 27.

20. Mejia A, López J, Uribe P. Detección de Candida en pacientes de prótesis total superior con diagnóstico clínico de estomatitis por prótesis Dental (EPD). Revista CES Odontología 1991: 4: 37.

21. Ceballos A. Micosis bucales. En: Ceballos A. Medicina Bucal. Grana. da: gráficas Anel; 1993. p.363 - 368.

22. Dinatale E. Respuesta alérgica en pacientes con estomatitis subprotésica y cultivo negativo para levaduras. URL Disponible en http:www.gacetadental.com.

23. Darwazseh G, Al-Refai S. Al-Mojaiwel S. Isolation of Candida species from the oral cavity and fingertips of complete denture wearers. J Prosthet Dent 2001: 86: 420 - 423.

24. Williams DW. Lewis MA. Isolation and identification of Candida from the oral cavity. Oral Dis 2000; 6: 3 - 11 .

25. Chandra J, Mukheriee PK, Leidich SD, Faddoul FF, Hoyer LL, Douglas LJ. Ghannoum MA. Antifungal resistance of candidal biofilms formed on denture acrylic in vitro. J Dent Res 2001; 80: 903 - 908.

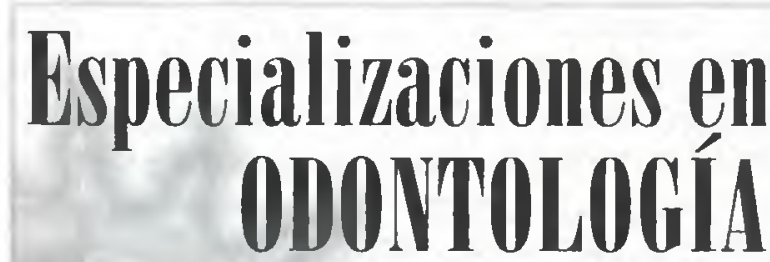

REHABILITACIÓN ORAL 。

170556170596800111100

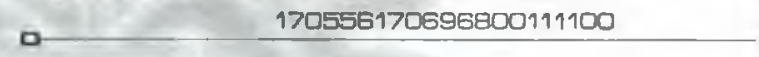

ODONTOPEDIATRÍA。 170556170616800111100

⿷.

ENDODONCIAO 170556170636800111100

\section{ORTODONCIA 170556170646800111100}

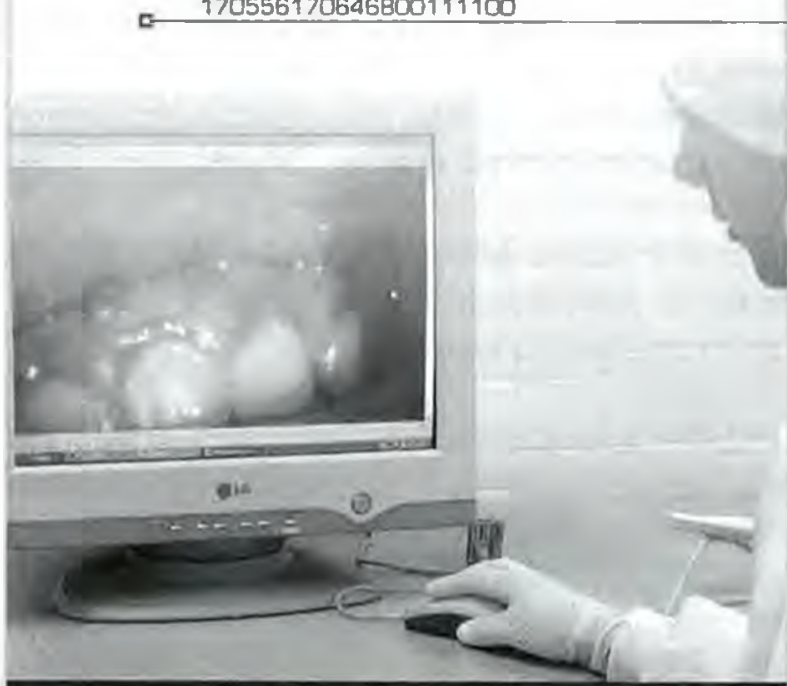

FACULTAD DE ODONTOLOGIA CAMPUS DE FLORIDABLANCA - EDIFECIO SANTANDOER www.ustahuca.edu.co 